Check for updates

Cite this: RSC Adv., 2018, 8, 15196

Received 8th February 2018

Accepted 6th April 2018

DOI: $10.1039 / \mathrm{c} 8 \mathrm{ra} 01252 \mathrm{k}$

rsc.li/rsc-advances

\section{Do two oxidants (ferric-peroxo and ferryl-oxo species) act in the biosynthesis of estrogens? A DFT calculation $\uparrow$}

\author{
Xiang-Yun Wang, ${ }^{a}$ Hui-Min Yan, ${ }^{a}$ Yan-Li Han, ${ }^{a}$ Zhu-Xia Zhang, (D) ${ }^{a}$ Xiao-Yun Zhang, ${ }^{a}$ \\ Wen-Jing Yang, ${ }^{a}$ Zhen Guo ${ }^{\text {D }}{ }^{* a}$ and Yan-Rong Li*b
}

Density functional theory calculations were performed in order to reveal the mysterious catalytic step of the biosynthesis of estrogens. The results indicated two reactive oxidants, ferric-peroxo and ferryl-oxo (compound I) species, to participate in the conversion of androgens to estrogens. The ferric-peroxo species was determined, according to our derived mechanism, to act in the oxidation of 19-OH androgen to yield the 19,19-gem-diol intermediate and generate the ferryl-oxo (compound I) species. This species was then modeled to effect, in the final step, an abstraction of $\mathrm{H}$ from an $\mathrm{O}-\mathrm{H}$ group of 19,19-gem-diol to give the experimentally observed products. We considered our new mechanistic scenario to reasonably explain the latest experimental observations and to provide deep insight complementing the newly accepted compound I (Cpd I) mechanism.

\section{Introduction}

Cytochrome P450 enzymes are ubiquitous hemoproteins that contribute to vital processes, such as drug metabolism, ${ }^{\mathbf{1 , 2}}$ the biosynthesis of steroids or lipids, and the degradation of xenobiotics., ${ }^{3,4}$ With a significant role in maintaining hormone balance, ${ }^{5}$ this aromatase has drawn considerable interest for its ability to construct an aromatic ring and as a promising inhibitor target in the treatment of hormone-dependent breast cancer. $^{6,7}$ Some of the crucial functions of cytochrome P450 include alkane hydroxylation, ${ }^{8}$ epoxidation, ${ }^{9}$ heteroatom oxidation, ${ }^{\mathbf{1 0}}$ and the biosynthesis of estrogen from androgen, the latter of which has been studied for a few decades. ${ }^{\mathbf{1 1 - 1 4}}$ Several mechanisms responsible for its final catalytic step have been proposed, both experimentally and theoretically, e.g., 4,5epoxidation, ${ }^{15}$ 2 $\beta$-hydroxylation, ${ }^{16}$ 10 $\beta$-hydroxyestr-4-ene-3,17dione formation, ${ }^{\mathbf{1 7}}$ ferric peroxide and compound I (Cpd I)mediated hydrogen abstraction, the latter two of which have become the most popular. For the ferric peroxide mechanism (Scheme 1(A)), a proton-assisted nucleophilic attack of the ferric peroxide intermediate onto C19 of the aldehyde group was theoretically calculated by Hackett et $a .^{13}$ to support the

${ }^{a}$ College of Material Science \& Engineering, Key Laboratory of Interface Science and Engineering in Advanced Materials, Ministry of Education, Taiyuan University of Technology, Shanxi, 030024, P. R. China.E-mail: guozhen@tyut.edu.cn

${ }^{b}$ Department of Earth Sciences and Engineering, Taiyuan University of Technology, Shanxi 030024, P. R. China.E-mail: li.dennis@hotmail.com

$\dagger$ Electronic supplementary information (ESI) available: Additional computational results, energies, and Cartesian coordinates of the optimized structure. See DOI: 10.1039/c8ra01252k experimental finding of the incorporation of ${ }^{18} \mathrm{O}$ atoms into the produced formic acids reported by Akhtar et al. ${ }^{12}$ Recent experimental studies, including resonance Raman spectroscopy and kinetic isotope effect ${ }^{18} \mathrm{O}$-labeling with high-resolution mass spectrometry, have indicated that $\mathrm{Cpd} \mathbf{I}$ as a reactive species could be involved in the final step of the production of aromatized estrogens from androgens, ${ }^{7,18}$ with ${ }^{18} \mathrm{O}$ exclusively incorporated into the water, rather than into the formic acids (Scheme 1(B)).

More recently, the above Cpd I mechanism was further supported by the theoretical works of Hirao et al., showing that Cpd Imediated hydrogen abstraction of the $\mathrm{O}-\mathrm{H}$ bond of 19,19-gem-diol has the exclusive low-energy-barrier channel to access the formic acid, the half-aromatized product and ${ }^{18} \mathrm{O}$-incorporating water. ${ }^{19}$ This conclusions of this work were in contrast to the previous

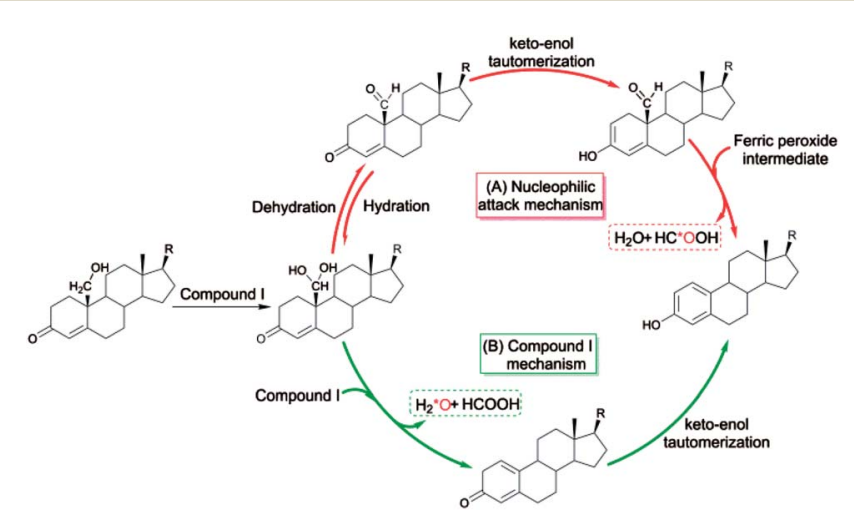

Scheme 1 (A) Nucleophilic attack mechanism. (B) Compound I mechanism. 
concepts and provided an unprecedented understanding of the third step of aromatase-catalysed estrogen formation. However, in light of the recent experiments of Guengerich and co-workers who employed 19-aldehyde as the starting substrate, the hydration of 19-aldehyde is now considered a prerequisite for the abstraction of hydrogen from the 19,19-gem-diol substrate by Cpd I. Therefore, evaluating the feasibility of isomerization (hydration and dehydration) between 19-aldehyde and 19,19-gem-diol is highly important for providing evidence for the Cpd I mechanism proposed by Hirao et al. In particular, Guengerich reported the nonenzymatic hydration of the 19-aldehyde to the 19,19-gem-diol to be about 4fold faster than the enzymatic formation of estrogen from 19-OH androgen. ${ }^{\mathbf{1 4}}$ Thus, when Cpd $\mathbf{I}$ is the only active species, either the rate of hydroxylation of the second step or the rate of hydrogen abstraction of the third step would be expected to be lower. Otherwise, this issue leaves us with an open-ended question: is there another oxidative species that can function as Cpd I in the aromatase-catalysed formation of estrogen? Here we conducted a computational study with the aim of answering the question listed above and providing deeper insight complementing the newly accepted Cpd I mechanism.

\section{Computational details}

\section{Theoretical methods}

All calculations were performed using the G09 package. ${ }^{20}$ We carried out density functional theory (DFT) calculations, using the M06L function with a standard 6-311G(d) basis set for the geometry optimization. Each transition state was performed by the presence of one imaginary frequency. Intrinsic reaction coordination (IRC) calculations were used to verify the connections of the corresponding intermediates in the respective potential energy surface. ${ }^{21}$ Solvent effects were considered by carrying out singlepoint calculations on the gas-phase stationary points in water solvent with Solvation Model Density (SMD) continuum solvation model. ${ }^{22}$ The M06 functional with a $6-311+\mathrm{G}(\mathrm{d}, \mathrm{p})$ basis set (SDD basis set for $\mathrm{Fe}$ ) was used to calculate the solvation single-point energies because of its improved description of nonbonding interactions that provide more accurate energy information. ${ }^{23,24}$ Unless otherwise specified, all subsequently discussed energies refer to solvation free energy $\left(\Delta G_{\mathrm{sol}}, \mathrm{kcal} \mathrm{mol}^{-1}\right)$ values, which were estimated as $\Delta G_{\text {sol }}=\Delta E_{\text {sol }}(\mathrm{SMD})+\Delta G_{\text {correction }}$ (gas), where $\Delta E_{\text {sol }}$ refers to the calculated solvation single point energy and $\Delta G_{\text {correction }}$ refers to the calculated thermal correction in the gas phase to the Gibbs free energy. To reduce the overestimation of the entropy contribution of the results, a correction of -2.6 (or 2.6) $\mathrm{kcal} \mathrm{mol}^{-1}$ for $2: 1$ (or $1: 2$ ) transformations was considered. Similar corrections have been deployed in other computational studies. ${ }^{25,26} 3 \mathrm{D}$ structures for the optimized stationary points were prepared with CYLview. ${ }^{27}$ Additional computational results are shown in the ESI. $\dagger$

\section{Model systems}

P450 catalyses the formation of estrogens from androgens, which are quite large substrates. In this study, we simplified the model

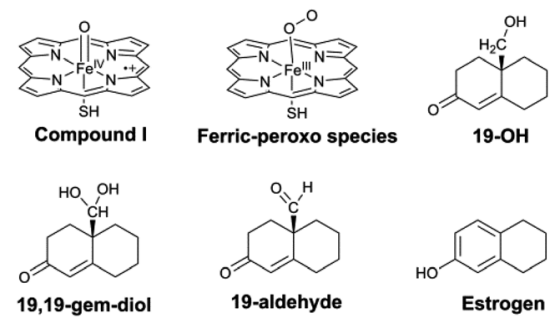

Fig. 1 Model systems used in this study.

of reactants and oxidant species of the P450 enzyme, as shown in Fig. 1.

\section{Results and discussions}

In order to assess whether the Cpd I species could be a unique active species for the formation of estrogen, we first investigated the water-assisted isomerization between 19-aldehyde and 19,19-gem-diol (III-1 to III-2, Scheme 2).

Water molecules play a significant role in many catalytic reactions. ${ }^{28-31}$ Experimental outcomes reported by Yoshimoto and Guengerich showed the presence of the 19-aldehyde and 19,19-gem-diol in a ratio of $1.0: 1.5$ in $\mathrm{D}_{2} \mathrm{O}$ at a $\mathrm{pH}$ of 7.8 . $^{\mathbf{1 4}}$ Inspired by this finding, herein, we decided to add water molecules that can actively participate in the isomerization of 19-aldehyde to 19,19-gem-diol. As shown in Fig. 2, the water molecules explicitly added into the reaction system had a significant effect on the hydration of 19-aldehyde to 19,19gem-diol. This effect was reflected by the fact that the barrier to the conversion of 19-aldehyde to 19,19-gem-diol decreased as the number of water molecules added was increased. That is, upon increasing the number of water molecules from one to three, the barrier to the reaction decreased from $42.6 \mathrm{kcal} \mathrm{mol}^{-1}$ to $21.9 \mathrm{kcal} \mathrm{mol}^{-1}$. This decrease could be ascribed to the water molecules serving as the $\mathrm{H}$ shuttles, markedly reducing the ring repulsion of transition states like (TS) $)_{3}$ with an eightmembered-ring transition state. All attempts to locate the transition states with four water molecules were unsuccessful. We envisioned the barriers to the hydration reactions assisted by more water molecules to not change too much in energy, similar to the calculated findings reported by Phillips et al. ${ }^{28,29} \mathrm{It}$ is worthwhile noting that the conversion of the 19-aldehyde to the 19,19-gem-diol in biological conditions is very different from nonenzymatic hydration reaction counterpart and could be

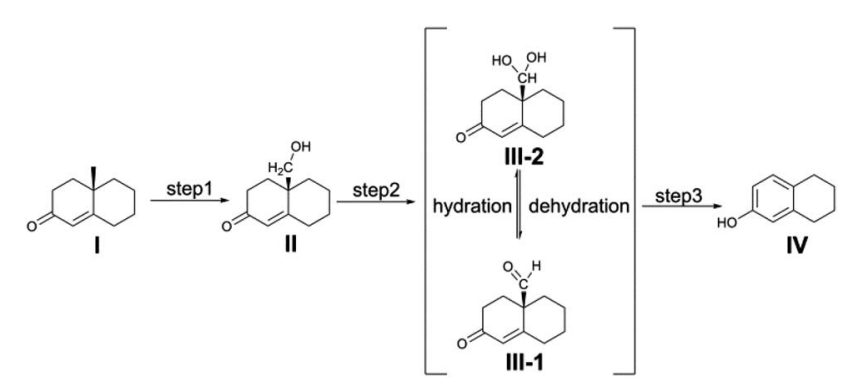

Scheme 2 Proposed oxidative steps of the conversion of androgens to estrogens catalyzed by cytochrome P450 19A1. 


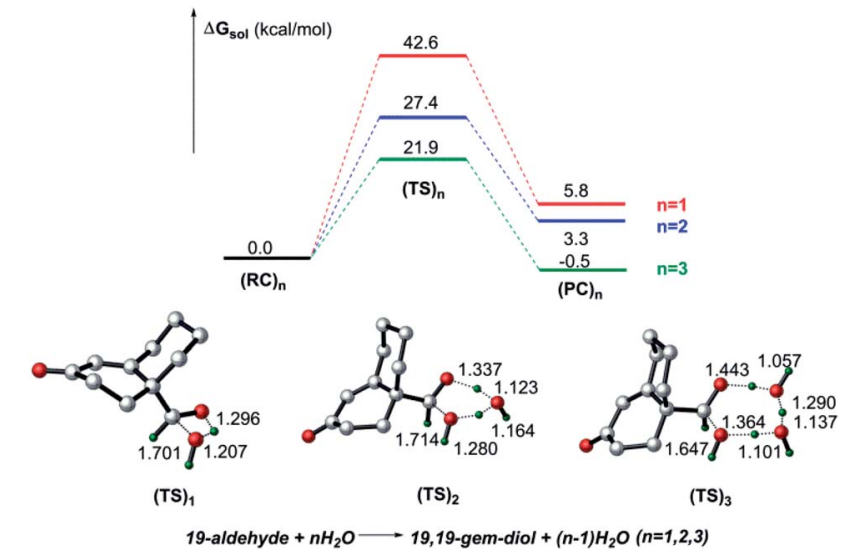

Fig. 2 DFT-calculated energy diagram for the isomerization between 19-aldehyde and 19,19-gem-diol, showing the computed structures of the key transition states, and selected bond distances $(\AA)$.

a rapid process. Unfortunately, there is a lack of experimental results for the enzymatic hydration reaction. As such, further investigation with more reliable methodologies, such as QM/ $\mathrm{MM}$ simulations or QM dynamics, are needed to give more reliable results on the above hydration reactions.

We next performed theoretical calculations on catalytic steps 2 and 3 of the enzymatic estrogen formation from 19-OH androgen (II to IV, Scheme 2) mediated by two potential active species: ferric-peroxo and ferryl-oxo (Cpd I).

Fig. 3 shows the reaction energy profiles and the optimized structures of intermediates and transition states for the oxidation of 19-OH androgen by Cpd I species. Although the final catalytic step (step 3, Scheme 1) has previously been analyzed using QM/MM calculations by Hirao et al. ${ }^{19}$ for the convenience of making comparisons, we recalculated the oxidation of 19,19gem-diol by the Cpd I species at the same level of theory as was used for the calculation of isomerization between 19-aldehyde and 19,19-gem-diol.

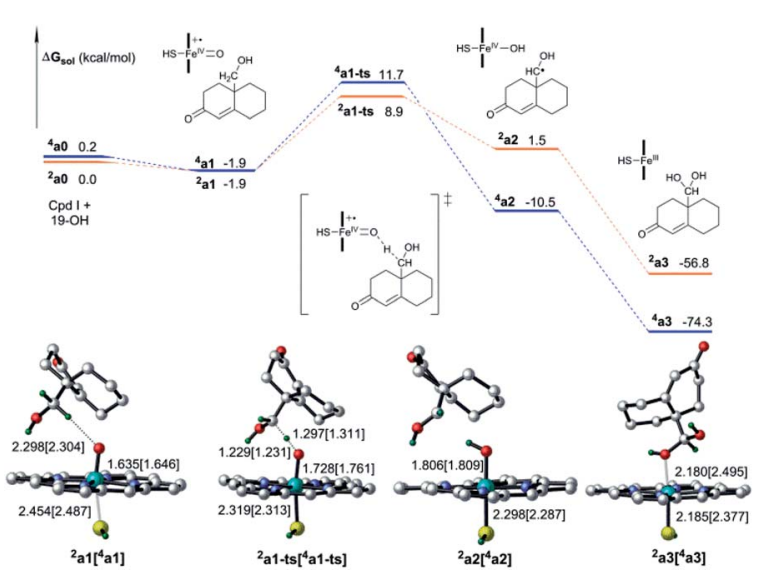

Fig. 3 DFT-calculated energy diagram for the oxidation of $19-\mathrm{OH}$ androgen by Cpd I species in doublet and quartet states. Shown are the computed structures of key transition states and intermediates, and selected bond distances $(\AA)$. The values outside of the parentheses are bond lengths in the doublet state, while those in the square brackets are in the quartet state.

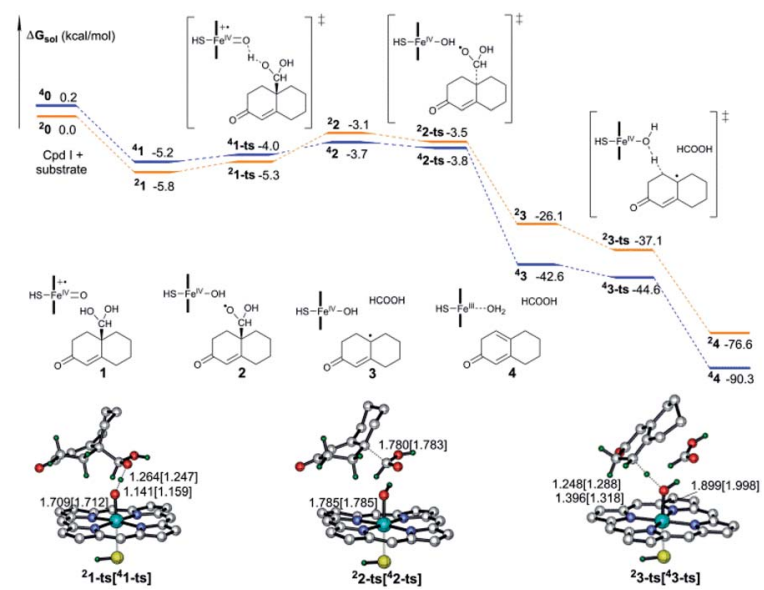

Fig. 4 DFT-calculated energy diagram for the oxidation of 19,19gem-diol by Cpd I species in doublet and quartet states; shown are the computed structures of key transition states, and selected bond distances $(\AA)$. The values outside of the parentheses are bond lengths in the doublet state, while those in the square brackets are in the quartet state.

As seen in Fig. 3 and 4, the reaction barriers to step 2 and step 3 to form estrogen catalysed by the Cpd I species, i.e., the hydroxylation of 19-OH androgen $\left(10.8 \mathrm{kcal} \mathrm{mol}^{-1}\right.$, Fig. 3) and the final catalytic step of the oxidation of 19,19-gem-diol (2.1 kcal mol ${ }^{-1}$, Fig. 4), were lower in energy than that of the nonenzymatic hydration of 19-aldehyde with three water molecules (21.9 kcal mol ${ }^{-1}$, Fig. 2), respectively. We considered this difference to be inconsistent with the recent experimental findings indicating the enzymatic formation of estrogen from 19-OH androgen (II to IV, Scheme 2) to be slower than the nonenzymatic hydration of 19-aldehyde. ${ }^{14}$ Therefore, Cpd I is unlikely to be the only active species in the formation of estrogen from 19-OH substrate. And we anticipated that another active species, one that may function as a Cpd I species, might participate in aromatase-catalysed estrogen formation. This issue was the key point that we aimed to highlight in this work.

The identity of oxidant species of cytochrome P450 enzyme in the conversion of androgen to estrogen remains poorly understood. ${ }^{32,33}$ To our knowledge, aside from the metal-oxo ${ }^{34,35}$ and hydroperoxo complexes ${ }^{36}$ and the $\mathrm{Fe}^{\mathrm{III}}\left(\mathrm{H}_{2} \mathrm{O}_{2}\right)$ complex, ${ }^{37}$ metalperoxo species has more recently received a lot of attention since it has been considered as an alternative reactive oxidant for $\mathrm{C}-\mathrm{H}$ bond activation of substrates in catalytic reactions of nonheme iron and copper enzymes. ${ }^{38-40}$ Herein, the involvement of the ferricperoxo species in the aromatization of androgen, which has been characterized using EPR and resonance Raman spectroscopy, ${ }^{41,42}$ was investigated computationally as shown in Fig. 5 .

Fig. 5 shows the activation of the $\mathrm{C}-\mathrm{H}$ bond mediated by the ferric-peroxo species. DFT calculations revealed the ferricperoxo-catalysed oxidation of $19-\mathrm{OH}$ androgen to start with abstraction of $\mathrm{H}$ from the $\mathrm{C}-\mathrm{H}$ bond of $19-\mathrm{OH}$ androgen, followed by a barrierless rebounding process of a hydroxyl group to the $\mathrm{C} 19$ radical. This transformation was determined to yield Cpd I and the intermediate b3 (Fig. 5). The latter has been 


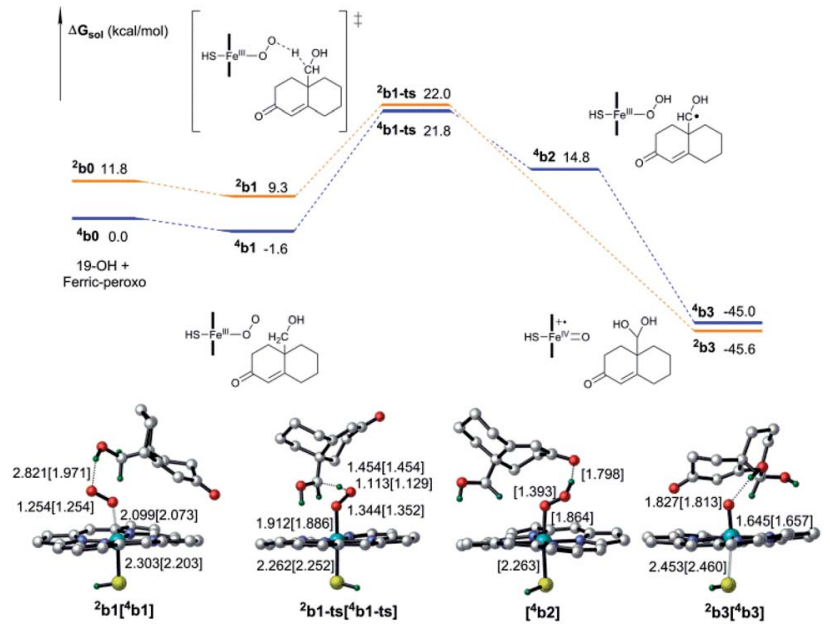

Fig. 5 DFT-calculated energy diagram for the oxidation of $19-\mathrm{OH}$ androgen by ferric-peroxo species in the doublet and quartet states; shown are the computed structures of key transition states and intermediates, and selected bond distances (Å). The values outside of the parentheses are bond lengths in the doublet state, while those in the square brackets are in the quartet state.

shown to be a necessary precursor for the newly accepted Cpd I mechanism (Scheme 1 and Fig. 4). ${ }^{19}$ Our calculation showed the abstraction of hydrogen by the ferric-peroxo species to require an activation free energy of $23.4 \mathrm{kcal} \mathrm{mol}^{-1}$ (Fig. 5), i.e., higher in energy than that of nonenzymatic hydration of 19-aldehyde. This calculation provided a rationale for the experimental result showing the nonenzymatic hydration of the 19-aldehyde to be more rapid than the enzymatic estrogen formation, indicating that the ferric-peroxo species might serve as a potential active oxidant in the conversion of $19-\mathrm{OH}$ androgen to 19,19 -gem-diol (step 2, Scheme 2).

An interesting feature was found regarding the oxidative conversion of 19-OH androgen (Fig. 3 and 5). Why is the reactivity of the ferric-peroxo species more variable than that of the Cpd I species? Frank Neese et al. showed the electronic structure and reactivity to be intimately linked. ${ }^{43}$ To gain more insight into the chemistry of the reaction mechanism, we carried out a natural bond orbital analysis of the frontier

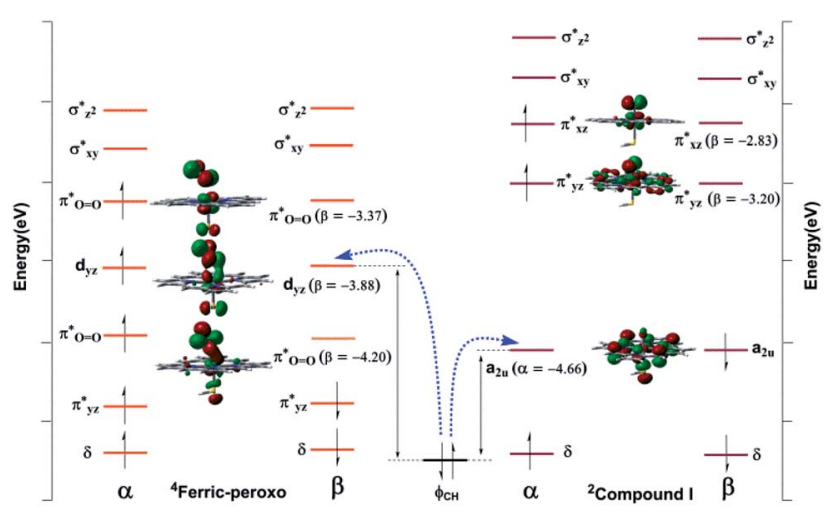

Fig. 6 The frontier molecular orbital (FMO) and electronic evolution for the activation of $19-\mathrm{OH}$ androgen via the Cpd I species in the doublet state (right) and ferric-peroxo species in the quartet state (left). molecular orbital pertinent to electronic evolution in the activation of the $\sigma_{\mathrm{CH}}$ bond.

Fig. 6 illustrates formally the electronic evolution for the oxidation of 19-OH androgen by two potential oxidants. The NBO analysis indicated the $\alpha$-electron, for the doublet state of the Cpd I species, to flow from the $\sigma_{\mathrm{CH}}$ bond of the substrate to the corresponding singly occupied $\mathrm{a}_{2 \mathrm{u}}$ orbital $(-4.66 \mathrm{eV})$ to fill the porphyrin radical cationic hole (Fig. 6, right)-but the $\beta$-electron of the $\sigma_{\mathrm{CH}}$ bond, in the case of the quartet state of ferric-peroxo species (Fig. 6, left), to move into the singly occupied $\mathrm{d}_{y z}$ orbital $(\beta$-LUMO $+1,-3.88 \mathrm{eV})$ of iron. Application of FMO theory implied the oxidation of 19-OH androgen mediated by the Cpd I species to require a lower reaction barrier due to the smaller energy gap between the partially occupied $\mathrm{a}_{2 \mathrm{u}}$ orbital of Cpd I species and the $\sigma_{\mathrm{CH}}$-HOMO of the substrate. This interpretation was in accordance with our calculated results (Fig. 3 and 5). On the other hand, inspection of the transition state structures of ${ }^{2}$ a1-ts and ${ }^{4}$ b1-ts showed a side-on attack mechanism, with such a side-on approach of the $\mathrm{C}-\mathrm{H}$ bond to the oxygen atom of ferryloxo and that of ferric-peroxo being best explained to be a result of optimizing steric considerations and orbital overlap. ${ }^{44}$ We also tried to locate the transition state with a vertical approach of the $\mathrm{C}-\mathrm{H}$ bond towards the porphyrin plane, which involved producing an overlap between the electron-accepting orbital $\left(\pi_{\mathrm{o}=0}^{*}, \beta\right.$-LUMO $)$ and electron-donating orbital $\left(\sigma_{\mathrm{CH}}\right)$. However, several trials failed to produce the desired stationary point.

As described above, hydroxylation and $\mathrm{H}$-abstraction catalysed by Cpd I were determined to have low reaction barriers $(10.8 \mathrm{kcal}$ mol $^{-1}$, Fig. 3 and 2.1 kcal mol $^{-1}$ Fig. 4), indicating Cpd I to unlikely be the sole oxidant in the formation of estrogen from 19-OH substrate. The ferric-peroxo species could be another potential active oxidant participating in the conversion of $19-\mathrm{OH}$ androgen to 19,19-gem-diol, due to the reaction barrier of the formation of 19,19-gem-diol from 19-OH androgen catalysed by ferric-peroxo species being higher than that of the nonenzymatic hydration of 19-aldehyde. This possibility is consistent with the experimental results showing the reaction constant of the enzymatic formation of estrogen from 19-OH androgen to be lower than that of the nonenzymatic hydration reaction of the aldehyde. In addition, would the ferric-peroxo species compete with the Cpd I species in the final catalytic step? Part of the DFT-computed energy profile is shown in Fig. 7 (the unabridged energy diagram is shown in Fig. S1†). This calculation revealed the reaction to occur via an

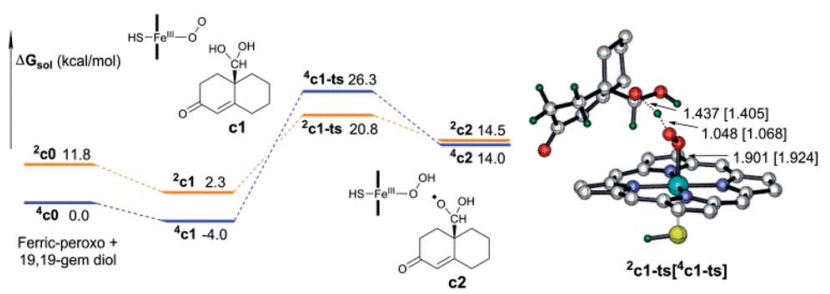

Fig. 7 DFT-calculated energy diagram for the aromatized reaction by ferric-peroxo species in doublet and quartet states; shown are the computed structures of key transition states, and selected bond distances $(\AA)$. The values outside of the parentheses are bond lengths in the doublet state, while those in the square brackets are in the quartet state. 


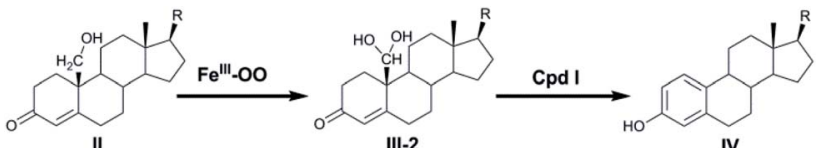

Scheme 3 The refined mechanism for the oxidation of $19-\mathrm{OH}$ androgen to estrogen.

abstraction of $\mathrm{H}$ from an $\mathrm{O}-\mathrm{H}$ bond of 19,19-gem-diol, which could not occur in the case of the Cpd I species due to the strikingly high reaction barrier of $24.8 \mathrm{kcal} \mathrm{mol}{ }^{-1}$ (compared to that $2.1 \mathrm{kcal} \mathrm{mol}^{-1}$ ). Taken together, for the conversion of androgen to estrogen, we concluded the 19-OH androgen to initially undergo hydroxylation catalysed by a ferric-peroxo species, and the generated Cpd I species to then participate in the final oxidative abstraction of $\mathrm{H}$ from 19,19-gem-diol (III-2, Scheme 3).

\section{Conclusions}

In summary, DFT calculations were conducted on the formation of estrogens from 19-OH androgen. The study revealed the ferric-peroxo species in conjunction with the Cpd I species to be responsible for the conversion of $19-\mathrm{OH}$ androgen to estrogen. The computations afforded the mechanism shown in Scheme 3, with this mechanism involving the initial reaction of the ferricperoxo species with the 19-OH androgen (II) to yield 19,19-gemdiol (III-2), followed by the Cpd I-catalysed abstraction of $\mathrm{H}$ from an $\mathrm{O}-\mathrm{H}$ bond of 19,19-gem-diol to give the products experimentally observed by Guengerich and co-workers. This work provided a further explanation for the recent experimental findings, and a deeper understanding of the newly accepted Cpd I mechanism.

\section{Conflicts of interest}

The authors declare no competing financial interest.

\section{Acknowledgements}

This work was supported by the 100-Talent Program in Shanxi Province, the Natural Science Foundation of Shanxi Province (No. 2015011034).

\section{Notes and references}

1 Y. Mie, M. Suzuki and Y. Komatsu, J. Am. Chem. Soc., 2009, 131, 6646-6647.

2 L. Ji, A. S. Faponle, M. G. Quesne, M. A. Sainna, J. Zhang, A. Franke, D. Kumar, R. van Eldik, W. Liu and S. P. de Visser, Chemistry, 2015, 21, 9083-9092.

3 M. C. Feiters, A. E. Rowan and R. J. M. Nolte, Chem. Soc. Rev., 2000, 29, 375-384.

4 I. Schlichting, J. Berendzen, K. Chu, A. M. Stock, S. A. Maves, D. E. Benson, R. M. Sweet, D. Ringe, G. A. Petsko and S. G. Sligar, Science, 2000, 287, 1615-1622.

5 D. Ghosh, J. Griswold, M. Erman and W. Pangborn, Nature, 2009, 457, 219-223.
6 R. W. Brueggemeier, J. C. Hackett and E. S. Diaz-Cruz, Endocr. Rev., 2005, 26, 331-345.

7 K. Sen and J. C. Hackett, Biochemistry, 2012, 51, 3039-3049.

8 A. Altun, S. Shaik and W. Thiel, J. Am. Chem. Soc., 2007, 129, 8978.

9 D. Kumar, S. P. de Visser and S. Shaik, Chemistry, 2005, 11, 2825-2835.

10 H. Hirao, D. Kumar, W. Thiel and S. Shaik, J. Am. Chem. Soc., 2005, 127, 13007-13018.

11 M. Akhtar, M. R. Calder, D. L. Corina and J. N. Wright, J. Chem. Soc., Chem. Commun., 1981, 3, 129-130.

12 M. Akhtar, M. R. Calder, D. L. Corina and J. N. Wright, Biochem. J., 1982, 201, 569-580.

13 J. C. Hackett, R. W. Brueggemeier and C. M. Hadad, J. Am. Chem. Soc., 2005, 127, 5224-5237.

14 F. K. Yoshimoto and F. P. Guengerich, J. Am. Chem. Soc., 2014, 136, 15016-15025.

15 H. Mastalerz and P. Morand, J. Chem. Soc., Perkin Trans. 1, 1982, 2611-2615.

16 E. Caspi, J. Wicha, T. Arunachalam, P. Nelson and G. Spiteller, J. Am. Chem. Soc., 1984, 106, 7282-7283.

17 E. Caspi, H. R. W. Dharmaratne and C. Shackleton, J. Chem. Soc., Chem. Commun., 1989, 1699-1700.

18 P. J. Mak, A. Luthra, S. G. Sligar and J. R. Kincaid, J. Am. Chem. Soc., 2014, 136, 4825-4828.

19 K. Xu, Y. Wang and H. Hirao, ACS Catal., 2015, 5, 4175-4179. 20 J. Mendes, K. J. de Almeida, J. L. Neto, T. C. Ramalho and H. A. Duarte, Spectrochim. Acta, Part A, 2017, 184, 308-317.

21 K. Fukui, Acc. Chem. Res., 1981, 14, 471-476.

22 Y. Chen, H. Zhang and Q. Liu, Spectrochim. Acta, Part A, 2014, 126, 122-128.

23 L. C. Burrows, L. T. Jesikiewicz, G. Lu, S. J. Geib, P. Liu and K. M. Brummond, J. Am. Chem. Soc., 2017, 139, 15022-15032.

24 J. M. Grandner, H. Shao, R. H. Grubbs, P. Liu and K. N. Houk, J. Org. Chem., 2017, 82, 10595-10600.

25 A. Ariafard, N. M. Ghohe, K. K. Abbasi, A. J. Canty and B. F. Yates, Inorg. Chem., 2013, 52, 707-717.

26 A. Ariafard, N. J. Brookes, R. Stranger and B. F. Yates, Organometallics, 2011, 30, 1340-1349.

27 C. Y. Legault, CYLview, 1.0b, Universite de Sherbrooke, 2009, http://www.cylview.org.

28 C. Zhao, X. Lin, W. M. Kwok, X. Guan, Y. Du, D. Wang, K. F. Hung and D. L. Phillips, Chem.-Eur. J., 2005, 11, 1093-1108.

29 Z. Guo, C. Zhao, D. L. Phillips, E. G. Robertson and D. Mcnaughton, J. Phys. Chem. A, 2008, 112, 8561-8568.

30 P. S. Peters, D. Duflot, A. Faure, C. Kahane, C. Ceccarelli, L. Wiesenfeld and C. Toubin, J. Phys. Chem. A, 2011, 115, 8983-8989.

31 P. Greenzaid, Z. Luz and D. Samuel, J. Am. Chem. Soc., 1967, 89, 749-756.

32 S. Shaik, S. Cohen, Y. Wang, H. Chen, D. Kumar and W. Thiel, Chem. Rev., 2010, 110, 949-1017.

33 B. Meunier, S. P. de Visser and S. Shaik, Chem. Rev., 2004, 104, 3947-3980.

34 J. Rittle and M. T. Green, Science, 2010, 330, 933.

35 J. T. Groves, J. Inorg. Biochem., 2006, 100, 434-447. 
36 I. G. Denisov, T. M. Makris, S. G. Sligar and I. Schlichting, Chem. Rev., 2005, 105, 2253-2278.

37 B. Wang, C. Li, K. B. Cho, W. Nam and S. Shaik, J. Chem. Theory Comput., 2013, 9, 2519-2525.

38 E. G. Kovaleva and J. D. Lipscomb, Nat. Chem. Biol., 2008, 4, 186-193.

39 J. Cho, S. Jeon, S. A. Wilson, L. V. Liu, E. A. Kang, J. J. Braymer, M. H. Lim, B. Hedman, K. O. Hodgson, J. S. Valentine, E. I. Solomon and W. Nam, Nature, 2011, 478, 502-505.
40 J. Cho, R. Sarangi and W. Nam, Acc. Chem. Res., 2012, 45, 1321.

41 I. D. G. Macdonald, S. G. Sligar, J. F. Christian, M. Unno and P. M. Champion, J. Am. Chem. Soc., 1999, 121, 376-380.

42 S. L. Gantt, I. G. Denisov, Y. V. Grinkova and S. G. Sligar, Biochem. Biophys. Res. Commun., 2009, 387, 169-173.

43 S. Ye, C. Y. Geng, S. Shaik and F. Neese, Phys. Chem. Chem. Phys., 2013, 15, 8017-8030.

44 J. T. Groves and T. E. Nemo, J. Am. Chem. Soc., 1983, 105, 5786-5791. 